\title{
Illuminating Awareness: Implications of fMRI Research in Disorders of Consciousness
}

The term "disorders of consciousness" (DOC) is applied to medical conditions that impair or abolish consciousness. Because coma-in which patients are both unarousable and lack awareness -is usually a transient state, patients with chronic DOC have as their principal feature impairment of awareness. ${ }^{1}$ Awareness has multiple components that can be variably affected: sensation; perception; memory; language function; emotion; cognition; metacognition; judgment; planning; motivation; societal functioning; and the ability to select or attend to various functions. ${ }^{2}$ DOCs include the vegetative state (VS), ${ }^{3}$ also called the unresponsive wakefulness syndrome (UWS $)^{4}$, in which there is wakefulness without awareness and minimally conscious state (MCS) in which only isolated aspects of awareness are preserved. ${ }^{5}$

The clinical assessment of DOC patients is difficult and depends on subjective interpretation of observed behavior. Indeed, the misdiagnosis rate among VS/UWS and MCS patients may be as high as $41 \%{ }^{6}$ Complicating matters further, it possible that a behaviorally unresponsive patient may retain covert conscious awareness. Indeed, a recent study found that $17 \%$ of patients accurately diagnosed in VS/ UWS using clinical diagnostic criteria ${ }^{3}$ exhibit volitional cognitive responses with functional magnetic resonance imaging (fMRI) and are, therefore, conscious. ${ }^{7}$ Thus, clinical criteria alone may not always be sufficient for the reliable determination of VS/UWS. Additionally, the diagnostic criteria for $\mathrm{MCS}^{5}$ may not be sensitive to the range of impairment in awareness.

\section{How CAN FMRI EXPLORE CONSCIOUS AWARENESS?}

Since 1991, when the first maps of human brain activation were obtained using fMRI, ${ }^{8}$ there have been almost 380,000 publications using fMRI methods for studying human brain networks. In spite of this formidable collection of scientific work, the sole clinical diagnostic application of fMRI is presurgical mapping of eloquent cortex. The major obstacle for developing a clinically diagnostic application using fMRI is the need to a generate brain activation map that is, in and of itself, a definitive diagnostic signature. Not only must this map represent a unique diagnostic network with high sensitivity and specificity, but also it must be obtainable in a single patient. To date, fMRI has shown this capability only when data from multiple subjects has been merged before analysis. fMRI images obtained from single subjects are inherently noisy and data averaging from multiple subjects becomes necessary to extract signals that reveal signature networks.

The solution to this problem, however, may not be as difficult as it seems in DOC patients. One reason for this is that defining a "consciousness" network is not essential. What is essential is the ability to define two functional networks that have distinct maps with spatial signatures such that there is no difficulty in distinguishing between them. Monti and colleagues use a mental imagery task in fMRI in which patients are instructed to imaging playing tennis or to move from room to room in their house. ${ }^{7}$ Hemodynamic changes in motor or spatial navigation areas (respectively) were unique to volitionally imagining these activities and indistinguishable from responses in healthy controls. The mental imagery task has been modified to allow for communication with highly select VS/UWS and MCS patients. Patients are asked a question and instructed to imagine playing tennis if their answer is "yes," and to imagine walking from room to room in their house is their answer is "no." Most recently, a patient who had a VS/MCS diagnosis for 12 years was able to answer a series of questions, including "Are you in pain?",

\section{FUTURE APPLICATION OF FMRI IN DOC}

Potential clinical applications of fMRI in DOC patients are considerable. In the future, fMRI may be used to improve diagnostic accuracy and provide valuable prognostic information. The prospect of communication with select patients opens the door to exploration of quality of life and even involvement in treatment decisions.

In this issue, Lee and colleagues explore the ethical implications of fMRI research into patients with chronic DOC. ${ }^{10}$ The authors conducted structured interviews with neuroscientists, medical practitioners, ethicists, and lawyers for their perspectives on clinical applicability and "ethical, legal and social consequences within the Canadian health environment." Among participants "there was general consensus that this technology has the potential for improving the quality of care for these vulnerable patients." 10 However, a variety of issues, including validation of methods, criteria for patient selection, prognostic value, and impact on the health system, needs to be dealt with before clinical adoption.

Lee and colleagues' study has usefully highlighted both the potential clinical impact of fMRI as well as the need for further research. We agree that further research is required in a number of areas. First, the evidence base supporting the use and reliability of fMRI in DOC patients' needs to be expanded. To date, fMRI studies in this patient population have involved relatively small number of patients. Larger patient cohorts are needed to develop robust estimates of the prevalence of preserved cognitive function in DOC patients. Patient factors, such as age, mechanism of brain injury, clinical findings, and other neuroimaging findings, correlated with covert awareness may usefully guide the development of clinical guidelines. Further evidence on fMRI and patient outcome would inform both clinical care and decision-making by family members.

ReCEIVED January 26, 2015. Final ReVisions SubmitTEd JANUARY 29, 2015. 
Second, consensus guidelines on the appropriate use of fMRI in DOC are needed to ensure the responsible adoption of this technology. As Lee and colleagues rightly note, media coverage of recent dramatic cases may lead to unrealistic expectations and even inappropriate demands for access to the technology. Responsible clinical use must be guided by the best evidence and appropriate stewardship of scarce resources.

Third, further research is needed to explore the interests and experiences of DOC patients. Very little is known about the subjective experience of DOC patients. Despite this, physicians and members of the public have strong intuitions about VS/UWS. ${ }^{11}$ The prospect of communication with select patients provides an opportunity to explore the lived experiences of these patients. The development of quality-of-life instruments suitable for use with fMRI could usefully guide treatment decisions and positively impact patient care. ${ }^{12}$

Fourth, protocols for the assessment of decision-making capacity using fMRI are required. Communication raises the prospect of involving patients in their own medical decisions. Before patients are asked about treatment preferences, however, we must have reliable and standardized means to assess their decision-making capacity. ${ }^{12}$ The challenges to capacity assessment are formidable as patients can currently only be asked a handful or "yes" or "no" questions in a single scanning session. A successful approach will both need to demonstrate that a patient has intact cognitive function required for decision making generally (e.g. memory, reasoning) and that he or she understands and appreciates the particular decision at hand. Although considerable further research is required, it may prove to be the case that some patients can make low- or mediumstake decisions regarding their own care.

fMRI offers a unique opportunity for enhancing diagnosis and establishing communication with patients in whom all other methods have failed. The impact on patients and families is potentially quite significant. It would seem therefore that further investigation is clearly warranted.

\section{Disclosures}

C.W. and G.B.Y. collaborate with Adrian M. Owen on a Canadian Institutes of Health Research-funded project on the ethics of neuroimaging after serious brain injury.

\author{
David Mikulis \\ Department of Medical Imaging, University of Toronto, \\ Ontario, Canada
}

Charles Weijer

Rotman Institute of Philosophy, Western University, London,

Ontario, Canada

\author{
G. Bryan Young \\ Department of Clinical Neurological Sciences, Western \\ University, London, Ontario, Canada \\ E-mail: bryan.young@lhsc.on.ca
}

\section{REFERENCES}

1. Posner JB, Saper CB, Schiff N, Plum F. Plum and Posner's stupor and coma. Oxford: Oxford University Press; 2007. P. 8.

2. Lavie N, Beck DM, Konstantinou N. Blinded by the load: attention, awareness and the role of perceptual load. Philos Trans R Soc Lond B Biol Sci. 2014;369:20130205.

3. The Multi-Society Task force on PVS. Medical aspects of the vegetative state. N Engl J Med. 1994;330:1499-508.

4. Laureys S, Celesia GG, Cohadon F, et al. Unresponsive wakefulness syndrome: a new name for the vegetative state or apallic syndrome. BMC Med. 2010;8:68.

5. Giacino JT, Ashwal S, Childs N, et al. The minimally conscious state: definition and diagnostic criteria. Neurology. 2002;58:349-53.

6. Schnakers C, Vanhaudenhuyse A, Giacino J, et al. Diagnostic accuracy of the vegetative and minimally conscious state: clinical consensus versus standardized neurobehavioral assessment. BMC Neurol. 2009;9:35.

7. Monti MM, Vanhaudenhuyse A, Coleman MR, et al. Willful modulation of brain activity and communication in disorders of consciousness. N Engl J Med. 2010;362:579-89.

8. Belliveau JW, Kennedy DN Jr, McKinstry RC, et al. Functional mapping of the human visual cortex by magnetic resonance imaging. Science. 1991;254:716-9.

9. Fernández-Espejo D, Owen AM. Detecting awareness after severe brain injury. Nat Rev Neurosci. 2013;14:801-9.

10. Lee G, Byram AC, Owen AM, et al. Canadian perspectives on the clinical actionability of neuroimaging in disorders of consciousness. Can J Neurol Sci. 2015;42:96-105.

11. Gray K, Knickman TA, Wegner DM. More dead than dead: perceptions of persons in the persistent vegetative state. Cognition. 2011;121:275-80.

12. Weijer C, Peterson A, Webster F, et al. Ethics of neuroimaging after serious brain injury. BMC Med Ethics. 2014;15:41. 